\title{
Massage provision by physiotherapists at major athletics events between 1987 and 1998
}

\author{
S D R Galloway, J M Watt
}

Br J Sports Med 2004;38:235-237. doi: 10.1136/bjsm.2002.003145

Background: The equivocal findings in the literature on efficacy of massage makes it difficult to assess the requirement for, or justify the use of, specialist massage personnel at major athletics events. However, the use of massage by athletes during training and competition remains popular.

Objectives: To quantify the amount of their time that physiotherapists devote to massage treatment at major athletics events in an attempt to determine the importance of this treatment modality, and to examine whether the use of massage at athletics events is changing over time.

Methods: Data recorded by the head team physiotherapist from 12 major athletics events (national and international events) between 1987 and 1998 were examined. For each event, the data included: total number of treatments administered by the physiotherapist, the treatment modalities used, and the number of attendances for treatment. The amount of massage provided was expressed as a percentage of the total number of treatments for each athletic event, and the pattern of change in use of massage treatment over time was evaluated.

Results: The percentage of time spent providing massage treatment ranged from $24.0 \%$ to $52.2 \%$ of the total number of treatments made. The overall median percentage of total treatments in the form of massage was $45.2 \%$. No significant increase or decrease in the use of massage as a treatment modality was observed between 1987 and 1998 in the athletics events examined ( $p=0.95$ ).

Conclusions: A significant proportion of physiotherapists' time is devoted to the delivery of massage treatment at athletics events. The demand for massage treatment has been steady over the time period, in the events for which data are available, indicating a consistent use of this treatment modality. Given the popularity of massage among athletes, consideration should be given to the use of specialist sports massage staff at major athletics events. Furthermore, it would seem prudent to further investigate the efficacy of the treatment.

$\mathrm{P}$ revious literature on massage and sports performance has focused on a wide variety of potential benefits to athletes. These have ranged from reducing pain perception and muscle soreness after activity to recovery of muscle strength, yet there is still conflicting evidence in most of these areas with little support for physiological benefits of massage. ${ }^{12}$ Despite the equivocal nature of the research, there is much anecdotal evidence, and continued belief of beneficial effects of massage by both athletes and physiotherapists.

Since the recommendation for further evaluation of the use of massage by Callaghan, ${ }^{3}$ no work has quantified its role in the treatment of athletes at competitive events. This study aimed to examine data on the amount of massage administered at major athletics events over an 11 year period in an attempt to quantify the importance of massage in the treatment/support of competitive athletes. In addition, it aimed to determine whether the role of massage in treatment of athletes has changed over this period.

\section{METHODOLOGY}

Archived data were obtained for 12 major athletics events in which British or Scottish athletes were competing (table 1) between 1987 and 1998. A mixture of events was available from national to international level. These were the only ones for which data existed on the proportion of physiotherapists' time spent performing massage on track and field athletes. Thus, they represent all track and field athlete data available for analysis. All data were collated by the head physiotherapist for the British or Scottish athletes involved, and in all cases refer only to track, field, and road race components of athletics events, including hotel/village and trackside/venue treatments. The physiotherapy team was not the same in all events assessed except for the head physiotherapist who collated the data. On one occasion a sports masseur was included as part of the support team (Barcelona 1992). Data collated included total number of treatments administered, number of massage treatments administered, massage use before, during, and after competition (non-specific massage), massage use for specific treatments (specific massage), as well as number of attendances for treatment and other treatment modalities-for example, ultrasound, interferential, strapping-and areas treated-for example, calf, shoulder.

Descriptive statistics were prepared for presentation of the massage data, and a Pearson correlation coefficient analysis was used to determine whether an association was present between use of massage and the year/event. All data are

Table 1 Events for which archived physiotherapy data were recorded between 1987 and 1998

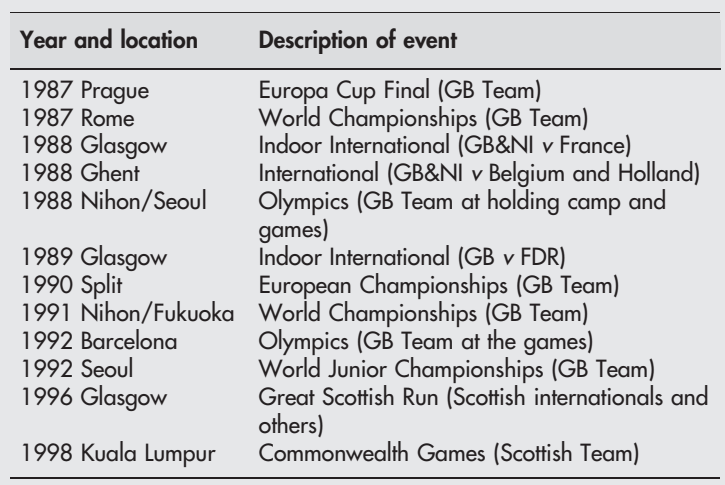




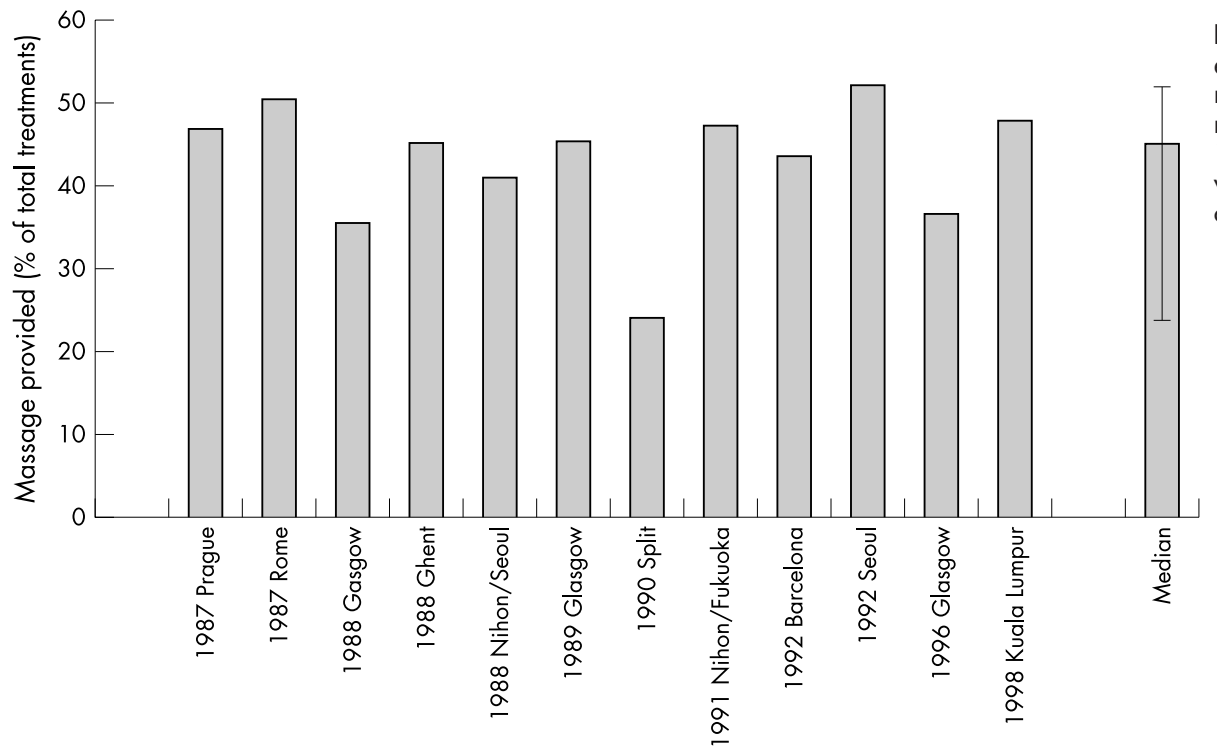

Figure 1 Massage provision expressed as a percentage of total number of treatments at each of the major athletic events studied between 1987 and 1998. Median and range values are shown for the combined data over the period assessed.

Year and location

expressed as raw values or median (range) where appropriate, and significance was taken at $\mathrm{p}<0.05$.

\section{RESULTS}

The archived data represent a range of competitive events from small national events to large international events incorporating over 4500 total treatments in an 11 year period. The total number of treatments administered at these events was 212 (44-1510) and the number of attendances for treatment was 49 (19-506). The number of treatments in the form of massage was 100 (18-619), and the number of ultrasound treatments was 19 (1-188). Figure 1 shows the percentage of total treatments administered as massage. Of these massage treatments, $27 \%$ were for specific problems associated with an injury and $73 \%$ were non-specific (before, during, and after competition). No clear change in the use of massage was evident over the time period assessed. In the one event in which a sports masseur was part of the support team, he administered 94 of 317 massage treatments.

The Pearson correlation coefficient for massage use, as a percentage of total treatments, with year was $0.02(\mathrm{p}=$ $0.95)$. A wide range of values is clearly observed from a minimum of $24.0 \%$ to a maximum of $52.2 \%$ (median $45.2 \%$ ) of total treatments being massage.

\section{DISCUSSION}

This analysis of archived data shows that a large amount of team physiotherapists' time is spent administering massage. In addition, it is clear that, despite a wide numerical range, the demand/use of massage at major athletics events has remained steady over the time period and events assessed. Furthermore, it has previously been indicated that massage represented $47 \%$ of all treatments administered to athletes from Great Britain over all sports in Atlanta 1996, ${ }^{1}$ and this fits with the data presented here. Given that a Great Britain team physiotherapist will work a scheduled eight hour shift every day, these data indicate that the physiotherapist could spend 1.9-4.2 hours (median 3.6 hours) providing massage treatment. When examined further, it is apparent that as much as three hours of the physiotherapist's time on each shift could be spent performing non-specific massage (before, during, and after competition).

These data raise four important points. Firstly, athletes and physiotherapists clearly feel that massage has a role to play in the management/treatment of the athlete at competitive events. It is clear that massage is largely used in the preparation for competition, between heats in competition, and in assisting recovery from competition rather than for treatment of specific problems. Secondly, it is unclear whether track and field athletes demand a greater amount of massage intervention than other athletes, or whether there is a preference for use of massage by particular physiotherapy teams. Therefore, it would seem appropriate for continued data collection to monitor the use of massage at major events, by sport, and to assess the use of massage by different physiotherapists. Thirdly, given the amount of time that physiotherapists devote to massage at major athletics events, it may be prudent to consider either a specialist sports masseur in the support staff for these events or a physiotherapist with postgraduate qualification in sports massage. Finally, without good quality, well controlled studies on the efficacy of massage before, during, and after competition, the use of massage remains wholly based on anecdotal accounts from athletes and physiotherapists. Although some studies are now being performed in this area, ${ }^{45}$ it is vital that more work is conducted to understand the mechanisms for the perceived benefits.

\section{Authors' affiliations}

S D R Galloway, J M Watt, Department of Sports Studies, University of Stirling, Stirling FK9 4LA, Scotland, UK

Correspondence to: Dr Galloway, Department of Sports Studies, University of Stirling, Stirling FK9 4LA, Scotland, UK;

s.d.r.galloway@stir.ac.uk

Accepted 25 February 2003

\section{REFERENCES}

1 Ernst $\mathrm{E}$. Does post-exercise massage treatment reduce delayed onset muscle soreness? A systematic review. Br J Sports Med 1998;32:212-14.

2 Tiidus PM. Manual massage and recovery of muscle function following exercise: a literature review. J Orthop Sports Phys Ther 1997;25:107-12.

3 Callaghan MJ. The role of masage in the mangement of the athlete: a review. Br J Sports Med 2000;27:28-33.

4 Monedero J, Donne B. Effect of recovery interventions on lactate removal and subsequent performance. Int J Sports Med 2000;21:593-7.

5 Hemmings B, Smith M, Graydon J, et al. Effects of massage on physiological restoration, perceived recovery, and repeated sports performance. $\mathrm{Br} J$ Sports Med 2000;34:109-15. 


\section{COMMENTARY}

In this very useful paper, the authors show that $24-52 \%$ of physiotherapists' time at major championships is taken up by massage. About $80 \%$ of such massage is "non-specific", coming into the category of "sports massage", for which physiotherapists are not specifically trained. Hence, if an appropriate complement of qualified sports masseurs could be taken with teams to major championships, it would free physiotherapists to concentrate more on their clinical work.
While noting the popularity of massage among competitors, the authors also emphasise the equivocal nature of research into its efficacy. Certainly the whole topic of massage, both as treatment and a pre-event "ergogenic aid", is a field ripe for more research into both its mechanisms and its efficacy-not least, perhaps, by sports psychologists. The authors are to be congratulated on a good attempt to quantify and categorise competitor demand for massage.

C Sharp 18 Salisbury Road, Moseley, Birmingham B13 8JS, UK; craig.sharp@brunel.ac.uk 